\title{
Solidão, um fator de risco
}

Ricardo Moreira Rodrigues*

\section{RESUMO}

A solidão constitui um problema social cada vez mais prevalente, devido ao envelhecimento populacional e às mudanças na organização da sociedade e na estrutura e dinâmica das famílias. A solidão é uma fonte de sofrimento significativo, associando-se à redução da qualidade de vida e ao aumento da morbilidade e mortalidade. Têm sido estudadas diversas intervenções dirigidas à redução da solidão, sendo mais eficazes as que abordam as cognições sociais maladaptativas. Torna-se urgente, numa sociedade cada vez mais envelhecida e isolada, a reflexão crítica sobre este tema, a sensibilização dos profissionais que lidam com este problema e o desenvolvimento de soluções que promovam uma sociedade mais integradora.

Palavras-chave: Solidão; Isolamento social; Suporte social.

A vida de relação é fundamental para o desenvolvimento do indivíduo, para a expressão da necessidade básica humana que é a comunicação e para o estabelecimento de relações interpessoais. Somos fundamentalmente uma espécie social e está na nossa natureza reconhecer, interagir e estabelecer relações com os nossos semelhantes. No entanto, a vida social exige uma série de tarefas, que nem sempre estamos capacitados para desempenhar da melhor forma, nomeadamente: 1) aprender por observação de contextos sociais; 2) reconhecer mudanças de status de amigos e inimigos; 3 ) comunicar através de linguagem verbal, paraverbal e não-verbal; 4) orquestrar relações, nomeadamente dentro do casal, na família, com amigos, grupos e coligações; 5) navegar através de hierarquias sociais complexas, normas sociais e evoluções culturais; 6) adaptar os interesses pessoais em prol do interesse do grupo social, com perspetiva de obter benefícios a longo prazo; 7) recrutar apoio para sancionar quem viola as leis do grupo; 8) fazer isto tudo numa gama de intervalos temporais, desde o passado distante até múltiplos futuros possíveis. ${ }^{1}$ Vivemos rodeados de pessoas, mas não necessitamos apenas da presença de outros; carecemos também da presença de pessoas que nos valorizem, em quem possamos confiar, com quem possamos comunicar, planear e trabalhar em conjunto. Não surpreende, portanto, que a investigação realizada nesta área tenha revelado que as relações sociais, em quantidade, mas especialmente

*USF Conde de Oeiras em qualidade, são importantes para manter o bem-estar físico e mental ao longo da vida. ${ }^{1}$

Um aspeto fundamental das relações humanas é o grau em que um indivíduo está socialmente isolado (isolamento social) ou se sente socialmente isolado (solidão). O isolamento social corresponde a um número reduzido de interações sociais e relacionamentos. Já a solidão, por sua vez, refere-se a um sentimento complexo, multidimensional e subjetivo resultante da perceção desagradável inerente à falta de apoio ou rede social. ${ }^{1-2}$

A solidão é determinada por fatores intrínsecos (como a personalidade) e por condicionantes extrínsecos (como a rede social de apoio). Com efeito, o indivíduo que se sente sozinho perceciona as relações sociais como insuficientes ou de baixa qualidade, tendo em conta as suas preferências de envolvimento social. ${ }^{2}$ Por conseguinte, é necessário considerar que existem pessoas que têm preferência por passar mais tempo sozinhas e ter uma rede social mais reduzida (isolamento ativo), sem implicar propriamente que se sintam sozinhas. Pessoas mais introvertidas têm preferência por baixos níveis de envolvimento social. Já a solidão implica uma discrepância entre as preferências pessoais de envolvimento social e a rede social que o indivíduo possui (isolamento passivo).

Tendo presente a distinção entre os dois conceitos acima expostos, acresce mencionar que existem diversas escalas que permitem medir a solidão. De um modo geral, incluem uma pergunta em que se solicita ao indivíduo estudado para responder se se sente sozinho ou 
se se tem sentido sozinho nos últimos tempos. Mais difícil é avaliar o grau em que o indivíduo se sente sozinho.

O suporte social é frequentemente relacionado com os conceitos de isolamento e solidão, significando ter família, amigos, profissionais de saúde ou outros a quem se possa recorrer em tempos de necessidade. ${ }^{1}$ Uma pessoa pode receber suporte, mas isto implicar uma relação de troca, um custo ou um sentimento de dívida que não ajudam a pessoa a sentir-se menos sozinha. Aliás, o suporte social pode não implicar a partilha de momentos bons ou provir de alguém com quem não se pretende ter uma ligação emocional, de tal ordem que os efeitos negativos da solidão se mantêm mesmo após ajustamento para a variável suporte social. ${ }^{1}$

Têm sido estudados diversos fatores protetores e fatores de risco para a solidão. Como fatores protetores identificam-se, designadamente, o casamento, a educação superior e o maior rendimento económico. ${ }^{1}$ Como fatores de risco identificam-se, nomeadamente, contactos pouco frequentes com amigos e família, uma rede social reduzida, o viver sozinho, a insatisfação com as condições de vida, a incapacidade, o stress laboral, o conflito familiar ou marital, as relações de baixa qualidade, o divórcio e a viuvez. ${ }^{1}$ Verifica-se maior prevalência de solidão no sexo feminino, diferença que se reduz após ajustamento para variáveis de confundimento como viuvez, depressão, problemas de mobilidade, idade, educação e rede social. ${ }^{3}$

A solidão é um problema prevalente e crescente. $\mathrm{O}$ aumento nas últimas décadas do isolamento e da solidão poderá dever-se ao envelhecimento populacional, ao aumento das taxas de divórcio, à maior mobilidade geográfica e à diminuição progressiva das dimensões das famílias.

A prevalência da solidão varia de acordo com a população estudada, o grupo etário e a escala utilizada para avaliar a solidão. Existe uma escala de solidão validada para a população portuguesa, a UCLA - Loneliness Scale, utilizada em diversos estudos. ${ }^{4}$

A nível europeu é aplicado regularmente o Inquérito Social Europeu que aborda diversas questões sociodemográficas e de atitudes e valores. ${ }^{5}$ Usando uma escala de solidão com a simples pergunta «...com que frequência ao longo da última semana lhe aconteceu sen- tir-se só?» e as categorias de resposta «Nunca ou quase nunca / Algumas vezes / A maior parte das vezes / Sempre ou quase sempre», avaliou-se a solidão em Portugal e nos restantes países europeus. Considerando o conceito de solidão frequente ou significativa para quem respondeu «A maior parte das vezes» ou «Sempre ou quase sempre», conclui-se que a prevalência de solidão em Portugal aumenta ao longo da idade, atingindo uma prevalência de $14,9 \%$ nas pessoas com mais de 60 anos. ${ }^{5}$ De notar que nos países nórdicos existe uma menor prevalência de solidão e não se verifica um aumento tão acentuado da mesma com o envelhecimento. Isto poderá dever-se a questões culturais, a políticas de envelhecimento ativo e a uma maior integração e participação dos idosos na sociedade. Já nos países do leste europeu verifica-se a maior prevalência de solidão, que poderá dever-se a questões históricas, a migrações populacionais e, reiteradamente, a diferentes aspetos culturais.

Como verificamos no Quadro I, a prevalência de solidão parece aumentar com a idade na maior parte dos países europeus estudados. A solidão nos idosos parece estar mais relacionada com as perdas sucessivas que ocorrem do que propriamente com o isolamento social. Estas perdas conduzem a uma redução progressiva do sentimento de pertença. ${ }^{6-7}$ Os filhos saem de casa, surgindo a síndroma do «ninho vazio», seguindo-se frequentemente a reforma, com a perda do papel laboral, do sentimento de se sentir útil e o afastamento da rede social laboral. Em países com políticas de envelhecimento ativo e valorização da população idosa promove-se a participação dos idosos na sociedade, permitindo que continuem integrados e com uma vida ativa, apesar de já não serem ativos laboralmente. Em Portugal, as políticas de envelhecimento ativo variam de região para região, sendo algumas bem-sucedidas, por exemplo, as que aproximam os idosos da população mais jovem. Contudo, são pouco personalizadas e a oferta é relativamente limitada. Em termos laborais existe também pouca flexibilidade para adaptação da carga de trabalho com a idade e o desemprego que, a partir dos 50 anos, é encarado como uma quase impossibilidade de regressar à vida ativa. As diminutas pensões de reforma e o aumento dos gastos em saúde acarretam um importante decréscimo de rendimento económico, que poderá limitar as atividades sociais que 


\begin{tabular}{|c|c|c|c|}
\hline \multicolumn{4}{|c|}{ Prevalência (\%) de solidão frequente } \\
\hline País & $<30 A$ & $30-59 A$ & $>=60$ \\
\hline Portugal & 6,5 & 9 & 14,9 \\
\hline Espanha & 4,4 & 6,5 & 11,5 \\
\hline França & 8,2 & 8,8 & 11,4 \\
\hline Áustria & 9,5 & 6,4 & 10,5 \\
\hline Reino Unido & 6,3 & 5,5 & 7,4 \\
\hline Suécia & 6 & 3,7 & 7,4 \\
\hline Holanda & 3,4 & 3,3 & 6 \\
\hline Noruega & 2,2 & 2,6 & 5 \\
\hline Alemanha & 5,1 & 4,4 & 7 \\
\hline Dinamarca & 3,4 & 1,9 & 3,2 \\
\hline Bulgária & 5,6 & 8,1 & 18,9 \\
\hline Ucrânia & 15,3 & 19,8 & 34 \\
\hline Russia & 11,3 & 15,4 & 24,4 \\
\hline Polónia & 5,5 & 11 & 20,1 \\
\hline
\end{tabular}

Fonte: Adaptado de Yang e Victor. ${ }^{5}$

os idosos têm oportunidade de realizar. Muitas atividades com envolvimento social têm um custo económico associado, o que poderá constituir uma barreira e um fator promotor de solidão nas pessoas com menor capacidade económica. Com o envelhecimento ocorrem ainda a perda dos amigos e do(a) companheiro(a). A perda do(a) companheiro(a) tem um impacto muito significativo. A pessoa com quem, muitas vezes, se criou uma vida em conjunto desaparece. O efeito é especialmente marcado nas pessoas com redes sociais mais reduzidas e que já apresentam alguma limitação. O declínio funcional que ocorre no envelhecimento é também bastante limitativo, tornando algumas pessoas «prisioneiras» da sua casa e conduzindo outras à institucionalização. O efeito da institucionalização na solidão varia de acordo com as características da instituição. Juntar pessoas pode não reduzir a solidão, especialmente se não existirem boas condições na instituição, se não existir terapeuta ocupacional, se os momentos sociais se limitarem à visualização de televisão e se o acesso da família for limitado.
A solidão é um sentimento com o qual todos nós já nos deparámos ao longo da vida. Este sentimento negativo pode ser útil, pois motiva a renovação das ligações sociais. Desde cedo, na nossa espécie, prosperámos vivendo em comunidade, sendo o isolamento um fator de risco para não sobrevivermos aos elementos adversos do meio que nos rodeia. Os sentimentos aversivos da solidão eram úteis para renovar as ligações necessárias para promover a sobrevivência, a coesão e a ação coletiva. A solidão pode ser encarada, deste modo, como um constructo biológico semelhante aos estímulos desagradáveis como a sede ou a dor física que são essenciais para se recuperar o equilíbrio homeostático e, no caso da solidão, a homeostasia social. ${ }^{1}$ No entanto, como frequentemente verificamos nas nossas consultas, também pode constituir uma fonte de grande sofrimento. Além disso, compromete o funcionamento executivo, o sono, a saúde mental e física. ${ }^{1}$ A longo prazo, estes efeitos traduzem-se num aumento da morbimortalidade. ${ }^{1,8}$

Tem-se verificado que sentimentos de solidão, quando mantidos ao longo do tempo, conduzem a uma ativação prolongada do eixo hipotálamo-hipófise-suprarenal, à semelhança do que acontece, por exemplo, na depressão. A par disso, o sujeito que se sente sozinho vai encontrar-se num estado de hipervigilância, especialmente em contextos sociais. Esta hipervigilância contribui para um enviesamento da cognição para os aspetos negativos do contexto social. Isto é, vai estar mais atento aos aspetos negativos da interação social e vai interpretar de modo negativo estímulos sociais neutros ou positivos. Estas cognições sociais que são maladaptativas influenciam os comportamentos e as interações sociais, dificultando o estabelecimento de novas relações, exacerbando o isolamento e os sentimentos de solidão. Assim, a solidão perpetua-se, tendo um impacto bastante significativo sobre o funcionamento social., ${ }^{2,7}$

A solidão associa-se a diminuição da qualidade do sono e a doenças psiquiátricas, como a depressão e a ansiedade. ${ }^{1-2,9}$ Verificam-se igualmente associações entre solidão e estilos de vida pouco saudáveis, como o tabagismo, consumo de álcool, sedentarismo e dieta pouco saudável, bem como com hipertensão, síndroma metabólica e doença cardiovascular. ${ }^{1,6}$ Os estudos são ainda insuficientes para se estabelecerem relações de causalidade e diversas hipóteses podem ser suscitadas para 
explicar estas associações. Sabe-se que a depressão é hoje em dia considerada um potencial fator causal de doença cardiovascular. A solidão poderá constituir um elemento a considerar nesta constelação de estilos de vida pouco saudáveis, doença psiquiátrica e doença cardiovascular. Serão necessários mais estudos para compreender o impacto das intervenções direcionadas para a solidão na saúde mental ou cardiovascular. Verifica-se ainda que os efeitos da solidão se poderão estender para além da morbilidade. Uma meta-análise de estudos longitudinais revelou uma probabilidade de mortalidade em $26 \%$ superior (Odds Ratio 1,26) nos indivíduos que se sentem sós. ${ }^{8}$ Isto poderá dever-se à associação de solidão com estilos de vida pouco saudáveis e doença cardiovascular. Por outro lado, a solidão está associada a um aumento do risco de ideação suicida ou de tentativas de suicídio. ${ }^{10}$ Esta relação é particularmente evidente nas pessoas com patologia psiquiátrica, mas também se observa na população geral.

Compreendem-se, portanto, os efeitos negativos em termos de bem-estar e saúde física, psicológica e social e a necessidade de desenvolver intervenções que reduzam a solidão.

Distinguem-se quatro tipos principais de intervenções na solidão: treino das capacidades sociais; aumento do suporte social; aumento das oportunidades de interação social e abordagem das cognições sociais maladaptativas. Masi e colaboradores realizaram uma revisão sistemática com meta-análise, integrando artigos com intervenções especificamente desenhadas para redução da solidão que tivessem medição quantitativa da solidão. ${ }^{11} \mathrm{~A}$ meta-análise dos estudos aleatorizados revelou uma pequena magnitude de efeito, mas significativa, das intervenções estudadas para redução da solidão. O tipo de intervenção que parece mais eficaz na redução da solidão é aquele em que se abordam as cognições sociais maladaptativas. ${ }^{11}$ As intervenções que se destinam a aumentar oportunidades para interação social através de atividades em grupo não são muito eficazes. Isto pode acontecer porque juntar pessoas isoladas não tende a criar novas amizades, dado que os pensamentos e os comportamentos dos indivíduos que se sentem sozinhos não os tornam atraentes para estabelecer novas relações.

O facto das intervenções que abordam as cognições sociais maladaptativas serem superiores em relação às demais é consistente com o modelo de solidão. A solidão acaba por se tornar um circuito regulador em que os indivíduos apresentam maior sensibilidade e vigilância em relação às potenciais ameaças de cariz social, tomam mais atenção aos aspetos negativos da informação social, relembram-se mais dos aspetos negativos dos eventos sociais e comportam-se de tal modo que não são socialmente atraentes, confirmando as suas piores expectativas. Este circuito tem características protetoras a curto prazo, mas a longo prazo aumenta a carga cognitiva, diminui o funcionamento executivo e influencia de modo adverso a saúde mental e física. De certo modo, a abordagem das cognições poderá intervir diretamente neste circuito regulador. Contudo, a abordagem das cognições sociais maladaptativas implica recursos humanos mais diferenciados, nomeadamente psicólogos, que, em Portugal, são claramente insuficientes. ${ }^{13}$

De referir que a meta-análise não inclui intervenções realizadas em Portugal. O efeito destas intervenções é modulado por diversos fatores, nomeadamente questões culturais, regionais e em termos de recursos que dificultam a sua extrapolação para a nossa realidade. É premente a realização de estudos em Portugal que avaliem a solidão e que avaliem a eficácia de intervenções que sejam facilmente generalizadas. De facto, existem muitas intervenções que promovem a redução do isolamento em Portugal, especialmente na população idosa, mas desconhece-se o seu efeito em termos de redução de solidão.

A solidão é um problema social e de saúde relevante, pois associa-se a morbimortalidade significativa e é, por si só, fonte de sofrimento e de redução da qualidade de vida. Não existem planos assistenciais, normas ou documentos que nos ajudem a abordar a solidão eficazmente. Torna-se urgente, numa sociedade cada vez mais envelhecida e isolada, a reflexão crítica sobre este tema, a sensibilização dos profissionais que lidam com este problema e o desenvolvimento de soluções que promovam uma sociedade mais integradora.

\section{REFERÊNCIAS BIBLIOGRÁFICAS}

1. Cacioppo JT, Cacioppo S. Social relationships and health: the toxic effects of perceived social isolation. Soc Personal Psychol Compass. 2014;8(2):58-72.

2. Hawkley LC, Cacioppo JT. Loneliness matters: a theoretical and empirical review of consequences and mechanisms. Ann Behav Med. 2010;40(2):218-27 
3. Richard A, Rohrmann S, Vandeleur CL, Schmid M, Barth J, Eichholzer M. Loneliness is adversely associated with physical and mental health and lifestyle factors: results from a Swiss national survey. PLoS One. 2017;12(7):e0181442.

4. Neto F.Avaliação da solidão da UCLA: adaptação portuguesa. Psicol Clin. 1989;2:65-79.

5. Yang K,Victor C:Age and loneliness in 25 European nations. Ageing Soc. 2011;31(8):1368-88.

6. Petitte T, Mallow J, Barnes E, Petrone A, Barr T, Theeke L. A systematic review of loneliness and common chronic physical conditions in adults. Open Psychol J. 2015;8 (Suppl 2):113-32.

7. Ong $A D$, Uchino $B N$, Wethington E. Loneliness and health in older adults: a mini-review and synthesis. Gerontology. 2016;62(4):443-9.

8. Holt-Lunstad J, Smith TB, Baker M, Harris T, Stephenson D. Loneliness and social isolation as risk factors for mortality: a meta-analytic review. Perspect Psychol Sci. 2015;10(2):227-37.

9. Cacioppo JT, Hughes ME, Waite LJ, Hawkley LC, Thisted RA. Loneliness as a specific risk factor for depressive symptoms: cross-sectional and longitudinal analyses. Psychol Aging. 2006;21(1):140-51.

10. Stickley A, Koyanagi A. Loneliness, common mental disorders and suicidal behavior: findings from a general population survey. J Affect Disord. 2016;197:81-7.
11. Masi CM, Chen HY, Hawkley LC, Cacioppo JT. A meta-analysis of interventions to reduce loneliness. Pers Soc Psychol Rev. 2011;15(3):21966.

12. Bekhet AK, Zauszniewski JA, Nakhla WE. Loneliness: a concept analysis. Nurs Forum. 2008;43(4):207-13.

13. Administração Central do Sistema de Saúde. Grupo de Trabalho para análise, estudo e elaboração de propostas relativamente aos modelos de organização da prestação de cuidados na área da psicologia no Serviço Nacional de Saúde: relatório final [Internet]. Lisboa: ACSS; 2017. Available from: https:/www.sns.gov.pt/wp-content/uploads/2017/08/ RelatorioFinalModelosOrganizacaoPsicologiaSNS.pdf

\section{CONFLITO DE INTERESSES}

O autor declara não possuir quaisquer conflitos de interesse.

\section{ENDEREÇO PARA CORRESPONDÊNCIA}

Ricardo Moreira Rodrigues

E-mail: ricardomoreirarodrigues@gmail.com

http://orcid.org/0000-0002-5899-4154

\section{Recebido em 03-04-2017}

Aceite para publicação em 21-06-2018

\section{ABSTRACT}

\section{LONELINESS, A RISK FACTOR}

Loneliness is a social problem with a growing prevalence, due to population ageing and changes in the organisation of society, and on family structure and dynamics. Loneliness is a cause of significant suffering, associated with a lower quality of life and with higher morbidity and mortality. A number of interventions aimed at reducing loneliness have been studied, and those approaching maladaptive social cognitions seem to be more effective.

In an increasingly aging and isolated society, it is urgent to critically discuss this issue, to raise awareness of professionals dealing with this problem, and to develop solutions that promote a more inclusive society.

Keywords: Loneliness; Social isolation; Social support. 